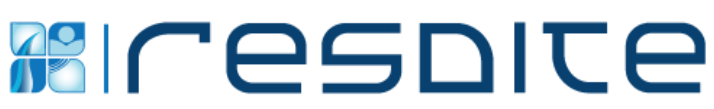

\section{A simulação como estratégia de formação de profissionais}

\section{da saúde}

\author{
Simulation as a training strategy for health professionals
}

\section{Karla Rona da Silva1, Sara Moura Martins ${ }^{2}$, Shirlei Moreira da Costa Farias ${ }^{3}$, Alanna Gomes da Silva ${ }^{4}$}

\section{Resumo}

Objetivo: O estudo teve como objetivo verificar se a participação no simulado sobre atendimento às vítimas de agravos em trânsito contribuiu para o aprimoramento dos conceitos, habilidades e conhecimento dos participantes. Métodos: A coleta de dados consistiu na abordagem das equipes, na qual foi entregue o questionário para o próprio participante responder. $O$ instrumento foi composto por questões relacionadas às características sociodemográficas e sobre a participação no simulado. Os dados obtidos nos questionários foram analisados utilizando o programa estatístico Data Analysis and Statistical Software (Stata), versão 13, por meio de estatística descritiva. Resultados: A avaliação final da atividade feita pelos participantes foi positiva e evidenciou satisfação frente à assimilação de conteúdo teórico e prático e também ao aprimoramento de habilidades e conhecimentos. Conclusão: Considerando suas potencialidades e fragilidades, vê-se diversos benefícios para os que utilizam a simulação como metodologia. Com isso, faz-se necessário um constante investimento em novas metodologias de ensino para que estudantes e profissionais absorvam cada vez mais conhecimento e, assim, cometam menos erros.

Palavras-Chave: Acidentes de Trânsito, Prevenção de Acidentes, Exercício de Simulação.

\begin{abstract}
Objective: The objective of this study was to verify participation in the simulation about attending to victims of transient diseases contributed to the improvement of participants' concepts, skills and knowledge. Methods: The data collection consisted of the teams approach, which was delivered the questionnaire to the respondent himself. The instrument was composed of questions related to sociodemographic characteristics and participation in the simulation. The data obtained in the questionnaires were analyzed using Statistical Data

\footnotetext{
1 Doutora. Universidade Federal de Minas Gerais - UFMG, Escola de Enfermagem. Email: karlarona0801@gmail.com 2 Aluna do Curso de Graduação em Gestão de Serviços de Saúde da Escola de Enfermagem, Universidade Federal de Minas Gerais. Email: sara_13moura@hotmail.com

3 Aluna do Curso de Graduação em Gestão de Serviços de Saúde da Escola de Enfermagem, Universidade Federal de Minas Gerais. Email: hirleidacosta16@gmail.com

4 Doutoranda no Programa de Pós-Graduação da Escola de Enfermagem, Universidade Federal de Minas Gerais. Email: alannagomessilva@gmail.com

Correspondência: Escola de Enfermagem UFMG, Av. Prof. Alfredo Balena, 190 - Santa Efigênia, Belo Horizonte - MG, Brasil. CEP: $30130-100$
}

Rev. Saúde Digital Tec. Educ., Fortaleza, CE, v. 5, n.1, p.04-15, jan./abr. 2020.

ISSN: 2525-9563 
Analysis and Statistical Software (Stata), version 13, using descriptive statistics. Results: The final evaluation of the activity performed by the participants was positive and showed satisfaction with the assimilation of theoretical and practical content and also improvement of skills and knowledge. Conclusion: Considering its potentialities and weaknesses, several benefits can be seen for those who use it as methodology. Thus, it is necessary to constantly invest in new teaching methodologies so that students and professionals absorb more and more knowledge and thus make fewer mistakes.

Key-words: Accidents Traffic, Accident Prevention, Simulation Exercise.

\section{Introdução}

No modelo tradicional de ensino é historicamente comum o uso de metodologias conservadoras, na qual o professor é o único responsável pelo aprendizado do aluno ${ }^{1}$. Nesse modelo, o aluno deve receber informações prontas e ter como única tarefa repeti-las na íntegra. No entanto, esse tipo de aprendizagem não é mais suficiente no mundo atual ${ }^{2}$, pois se torna fragmentada, e, por isso, tem sido substituída por métodos nos quais os estudantes praticam um papel mais efetivo em seu aprendizado. Nesse contexto, as metodologias ativas são processos nos quais os alunos se tornam responsáveis pela aquisição e assimilação de conteúdo em conjunto com o professor $^{3}$.

As metodologias ativas de aprendizagem vêm sendo sustentadas pela problematização e na evidência, ajudando os profissionais a resolverem seus desafios com a capacidade de inovação ${ }^{4}$. Assim, a problematização é pautada na observação de uma situação de modo crítico, buscando favorecer o vislumbre de diferentes realidades de um mesmo cenário. Essa estratégia motiva o discente a examinar um assunto, relacioná-lo e refletir acerca dele, promovendo a descoberta de novos significados ${ }^{3}$.

Dentre as metodologias ativas de aprendizagem tem-se a simulação, fundamentada na utilização da reprodução parcial ou total de um evento empírico em um modelo artificial. Ou seja, simula-se um acontecimento para que os envolvidos possam desenvolver suas habilidades naquela área, sejam elas manuais ou relacionadas às tomadas de decisões ${ }^{5}$.

O foco do ensino para a simulação é direcionado para habilidade técnica, com reincidência exaustiva do processo ${ }^{5}$. Para que a simulação seja bem-sucedida é necessário ter um conhecimento teórico breve, que, no caso, trata-se de todo conhecimento teórico adquirido previamente. Esse conhecimento é importante para que os participantes alcancem um melhor desempenho ao exercer suas funções durante a atividade ${ }^{6}$.

O aprendizado baseado em simulação tem se tornado um método de educação amplamente utilizado pelas 
instituições de ensino, sobretudo nos Estados Unidos e em países europeus, pois acredita-se no aprimoramento do conhecimento e do pensamento crítico dos alunos ${ }^{7,8}$. Por se tratar de uma plataforma de aprendizado com baixo risco, os alunos e profissionais podem se habituar aos procedimentos até que obtenham competência e domínio para realizá-los em um ambiente não controlado. Assim, quando aliado à educação em saúde, diminui-se a chance de erros e aumenta-se a segurança dos pacientes ${ }^{8}$.

A simulação também permite uma articulação entre a universidade, o serviço e a comunidade, devido a possibilidade de uma intervenção que consiste sobre a realidade, valorizando todos os atores que participam do processo e seus diferentes conhecimentos, promovendo também iniciativa no processo de pensar e o trabalho em equipe, não deixando de buscar modelos que ajudem a incorporar as práticas do sistema de saúde ${ }^{3,9}$. Assim, possibilita-se uma diferenciação do modelo de ensino tradicional, fazendo com que os alunos e profissionais tenham competência para reconstruir o saber e não simplesmente reproduzir o aprendizado de modo mecânico e acrítico ${ }^{9}$.

A prática simulada é uma tecnologia educacional não convencional utilizada com 0 intuito de promover um aprendizado significativo que alia os saberes teóricos aos práticos, propiciando aos alunos e participantes a possibilidade de construção de um pensamento crítico no que se relaciona a promoção e a prevenção de agravos à saúde ${ }^{7}$. Essa metodologia de aprendizagem ativa tem se tornado cada vez mais importante no meio didático e é considerada uma abordagem pedagógica intrínseca para uma boa formação universitária, pois colabora para a aquisição de experiência prática dos estudantes e profissionais de saúde ${ }^{3}$.

Nesse sentido, o estudo teve como objetivo verificar se a participação no simulado sobre atendimento às vítimas de agravos em trânsito contribuiu para o aprimoramento dos conceitos, habilidades e conhecimento dos participantes.

\section{Método}

Trata-se de um estudo transversal, descritivo, de natureza quantitativa, desenvolvido com 31 participantes do evento de extensão universitária que versa sobre uma simulação de atendimento às vítimas de acidente de trânsito. Foram incluídos no estudo os alunos regularmente matriculados no curso de graduação em Gestão de Serviços de Saúde de uma Instituição de Ensino Superior pública e excluídos os alunos que não cursaram a disciplina "Rede de Atenção as Urgências". Participaram também da pesquisa os profissionais que desenvolviam suas 
atividades laborais em serviços de atendimento pré-hospitalar no estado de Minas Gerais, Brasil. Foram incluídos profissionais do Serviço de Atendimento Móvel de Urgência (SAMU) e do Corpo de Bombeiros Militar de Minas Gerais (CBMMG) designados pelo Centro de Comando operacional dos respectivos serviços.

A simulação foi implementada como um evento de extensão universitário que visa operacionalizar um exercício prático que estimula a aprendizagem significativa. Assim, tem-se o discente como protagonista de toda ação, participando ativamente das fases de planejamento, execução e avaliação de todo processo, sob orientação de uma docente.

Para a ação foi montado um cenário, no estacionamento do campus universitário, simulando uma colisão frontal de um carro versus um ciclista, na sequência, o veículo desgovernado colidiu contra uma árvore, havendo duas vítimas politraumatizadas graves (o ciclista que ficou no solo inconsciente sobre a bicicleta e o condutor que ficou consciente dentro do automóvel, mas preso às ferragens).

Frente a essa simulação, os profissionais do SAMU e do CBMMG atuaram na avaliação da segurança e sinalização da cena, no desencarceramento (realizado exclusivamente por profissionais do CBMMG) e no atendimento conjunto às vítimas. Aos alunos incumbiu a organização e gestão de todo o evento. Ao término dessa etapa, todos os participantes dialogaram sobre as atividades realizadas como forma de compartilhamento, aprendizado e fortalecimento mútuo, principalmente discutindo sobre o trabalho em equipe e a comunicação verbal, em um formato do tipo Debriefing. Nesse momento ocorreu a coleta de dados para esta pesquisa, utilizando-se de um mesmo questionário adaptado do instrumento de Dourado e Giannella $^{10}$ com questões relativas às: expectativas, assimilações de conteúdo, habilidades e conhecimentos adquiridos durante o simulado. Vale destacar que este estudo tem como foco uma análise quantitativa das respostas apresentadas, assim, a ação extensionista foi descrita de forma detalhada para elucidar o contexto de aplicação do questionário. Todos os participantes foram esclarecidos sobre os objetivos da pesquisa, direito de recusa de participação, garantia do anonimato e relevância da assinatura do Termo de Consentimento Livre e Esclarecido (TCLE).

Os dados obtidos dos questionários foram analisados no programa estatístico Data Analysis and Statistical Software (Stata), versão 13, por meio de estatística descritiva. Nessa análise, os dados referentes ao perfil sociodemográfico foram expressos em percentagens. As respostas às questões que versaram sobre expectativas, assimilação, 
conceitos, habilidades e conhecimentos, foram representadas por categorias correspondentes: Muito pouco, Pouco, Regular ou Muito. As questões relativas à avaliação da atividade e qualidade do conhecimento foram categorizadas como Ruim, Regular, Bom ou Excelente.

Este trabalho atendeu a Resolução no 466/2012 do Conselho Nacional de Saúde que regulamenta a pesquisa com seres humanos e foi aprovado pelo Comitê de Ética em Pesquisa da Instituição de Ensino Superior responsável pelo estudo, CAAE $\mathrm{n}^{\circ}$ 94043218.0.0000.5149.

\section{Resultados}

Participaram do estudo 31 indivíduos, sendo 11 estudantes do curso Gestão de Serviços de Saúde e 20 profissionais. As demais características sociodemográficas encontram-se na Tabela 1.

Em relação à participação em atividades simuladas, $18 \quad(58,06 \%)$ participaram pela primeira vez e 13 $(41,94 \%)$ haviam participado em outros eventos.

Quanto à atividade realizada, $48,39 \%$ participaram da organização do evento e $45,16 \%$ participaram do atendimento direto às vítimas. No que se refere à avaliação dessas atividades realizadas, $19,35 \%$ avaliaram como boa e $80,65 \%$ como excelente.
$\mathrm{Na}$ questão sobre o que motivou para participação do simulado, poderia ser marcada mais de uma questão. As principais respostas foram: necessidade de aprendizado prático (26,09\%); aprimorar os conhecimentos acerca do tema (30,61\%); oportunidade de ver e perceber a logística e gerenciamento de um evento; e divulgação do trabalho realizado pelas equipes de saúde $(32,61 \%)$. De forma semelhante, foi perguntado sobre as dificuldades encontradas durante a realização da simulação, e os principais resultados foram: dificuldade de trabalhar em equipe $(6,06 \%)$ e dificuldade de tomar decisões importantes (3,03\%).

As questões temáticas relativas às expectativas, assimilações de conteúdo, habilidades e conhecimento adquirido, objetos principais de análise neste estudo, apresentaram resultados com frequências relevantes que foram descritos na Tabela 2.

No que se refere à avaliação da qualidade do conhecimento adquirido, $35,48 \%$ responderam que o conhecimento foi bom e $64,52 \%$ excelente.

Por fim, foi abordado sobre quais elementos da simulação foram favoráveis para aprendizagem, sendo essa uma questão aberta, na qual 30 participantes responderam, e as principais respostas foram: trabalho em equipe; procedimentos para a organização de um evento; aprendizagem sobre o funcionamento do 
sistema de urgência e emergência; o funcionamento de uma simulação e a importância de sua similaridade com a realidade; como proceder em casos de acidentes; a vivência prática de integração entre às equipes do SAMU e do CBMMG; alinhamento do conhecimento teórico com a prática; práticas de educação em saúde.

\section{Discussão}

Neste estudo foi possível avaliar a participação de estudantes nas etapas de planejamento, avaliação e gestão da atividade. Assim, o conceito metodológico da simulação foi potencializado, incluindo aspectos relativos não só ao contexto assistencial, mas também oportunizando a prática da gestão de recursos humanos, materiais, financeiros, logísticos e de infraestrutura.

De se ressaltar que o curso de Gestão de Serviços de Saúde tem por objetivo formar recursos humanos capazes de atuar de maneira inovadora, humanista e eticamente comprometida com as necessidades de saúde da população, por meio de ferramentas para identificar, diagnosticar e propor soluções criativas em áreas críticas, ampliando a capacidade de resposta dos serviços de saúde nos diferentes níveis de complexidade. Logo, é importante que o gestor seja crítico, empreendedor e tenha uma ampla visão do contexto social, político e econômico do país. Nesse sentido, esta atividade simulada permitiu aos discentes experimentar e fortalecer habilidades e competências que são essenciais à sua formação.

A educação em saúde tendo como base atividades de simulação é uma abordagem pedagógica útil, pois proporciona aos participantes a propriedade de tomada de decisão e aprimoramento de habilidades, de competências em experiências reais sem prejudicar a saúde de um paciente, como apresentado neste estudo. A educação em saúde é uma tarefa complexa que necessita de atenção e contínuo aprendizado, com investimento em novas metodologias de ensino para que estudantes e profissionais absorvam cada vez mais conhecimento e, assim, cometam menos erros ${ }^{4,11}$.

Torna-se importante que, durante sua formação acadêmica, o futuro profissional da saúde seja capaz de desenvolver habilidades para lidar com as mais adversas situações do dia a dia, sendo importante que ele saiba agir e se adaptar ao ambiente de trabalho, solucionando diversos problemas que aparecem no decorrer de suas atividades. Para que isso ocorra, necessita-se de três pilares essenciais: o conhecimento, a habilidade e a atitude ${ }^{12}$.

Nesse contexto, o simulado possibilita a integração multiprofissional e o trabalho em equipe, além de exercitar a organização, tomada de decisão, comunicação, bem como o gerenciamento 
de conflitos. O trabalho em equipe e a tomada de decisão foram descritas, pelos participantes desta investigação, como dificuldades encontradas durante a realização da simulação ${ }^{13}$.

Em nossa realidade, para os profissionais do CBMMG e do SAMU é bem comum a participação em atividades simuladas, mas para os alunos do Curso de Gestão de Serviços de Saúde se trata de uma atividade inovadora. Com efeito, a participação durante a organização e realização do evento se faz relevante para que os discentes exercitem o trabalho em equipe e aprimorem a tomada de decisão. Nessa perspectiva, aos alunos participantes foi oportunizado combinar estilos diferentes de abordagens educacionais de aprendizagem em seu processo de formação, sendo essa uma estratégia diferencial para consolidação do conhecimento e fortalecimento da autoconfiança.

A partir dos resultados obtidos com a presente pesquisa, entende-se que a realização de simulações no âmbito educacional e profissional da saúde é uma ferramenta que promove melhorias no desempenho dos participantes, pois permite a prática de ações e procedimentos em um ambiente de baixo risco, possibilitando o aprimoramento do conhecimento e das habilidades ${ }^{8,14,15}$.

A melhoria no processo de aprendizagem se deve, em grande parte, a memorização que acontece após algumas repetições do processo ${ }^{6,16}$. Dessa forma, este estudo reforça que é possível desconstruir as tarefas complexas e tornar o aprendizado mais dinâmico e eficiente a partir da simulação como metodologia de ensino e aprendizagem ativa.

Modelos de simulação funcionam como programas e ferramentas dinâmicas de suporte à decisão que integram diversas formas de evidências, como foi bem ratificado nesta investigação. Assim, o uso de tecnologias educacionais que minimizem possíveis restrições na aprendizagem, como é proposto pela prática simulada, é uma metodologia que facilita a conexão do conteúdo teórico com o prático e propicia uma menor chance de erros no âmbito profissional. Ao se tratar da área da saúde, as falhas profissionais, costumeiramente, culminam em prejuízos para os pacientes. Logo, o treinamento prévio de procedimentos possui um impacto positivo na melhoria dos resultados sobre conhecimento, habilidades e comportamento profissional ${ }^{17}$.

Estudos mostraram que a utilização e realização de atividades práticas em cenários reais facilitam a assimilação de conteúdo teórico, aprimoram conceitos e habilidades, 0 que favorece a aprendizagem significativa ${ }^{8,13,14}$.

A limitação identificada refere-se ao pequeno tamanho amostral decorrente do quantitativo de participantes da simulação. 
Contudo, buscou-se explorar com fidedignidade e rigor científico todos os resultados e análises para esta pesquisa.

\section{Conclusão}

O estudo mostrou que a participação na simulação como estratégia de ensino aprendizagem contribuiu para o aprimoramento dos conceitos, habilidades e conhecimento dos participantes. Ademais, foi possível confirmar que as expectativas frente à participação no simulado foram totalmente atendidas, assim como a melhor assimilação de conteúdo teórico. Os partícipes referiram, ainda, que esta estratégia potencializa a aprendizagem significativa com ampliação da qualidade para a formação acadêmica e capacitação profissional.

A simulação é uma importante ferramenta para o desenvolvimento e aprimoramento de competências inerentes aos profissionais da saúde. Isso posto, faz-se necessário um constante investimento em novas metodologias de ensino, como as simulações, para que estudantes e profissionais adquiram cada vez mais conhecimento e, assim, minimizem os erros em seu fazer laboral.

\section{Referências}

1. Protetti FH. Afinal, existe alguém aspecto positivo no modelo da Escola Tradicional? Revista Espaço Acadêmico. 2010; 9 (106): 75-83.

2. Santos JCF. A aprendizagem significativa passa pela avaliação formativa. 2015 [acessado 2019 Jul

09]. Disponível em:

http://juliofurtado.com.br/wp-

content/uploads/2015/08/Texto-A-apz-

significativa-passa-pela-

avalia\%C3\%A7\%C3\%A3o-

formativa.pdf

3. Mitre SM, Siqueria-Batista R, Girardide-Mendonça JM, Morais-Pinto NM, Meirelles CAB, Pinto-Porto Claudia, Moreira T, Hoffmann LMA.

Metodologias ativas de ensinoaprendizagem na formação profissional em saúde: debates atuais. Cien Saúde Colet 2008; 13 (Supl 2): 2133-2144.

http://dx.doi.org/10.1590/S141381232008000900018

4. Tanaka RY, Catalan VM, Zemiack J, Pedro ENR, Cogo ALP, Silveira DT. Objeto educacional digital: avaliação da ferramenta para prática de ensino em enfermagem. Revista Acta Paulista de Enfermagem 2010; 23 (5): 603-607. http://dx.doi.org/10.1590/S010321002010000500003

5. Pazin Filho A, Scarpelini S. Simulação: definição. Medicina 2007; 40 (2):162166.

6. Gogle J. Using Simulation-Based Learning to Prepare for a Potential Cardiac Emergency on the Labor Unit. Nursing for Women's Health 2017; 21(1): 20-27.

https://doi.org/10.1016/j.nwh.2016.12.0 09

7. Ferreira C, Carvalho JM, Carvalho FLQ. Impacto da metodologia de simulação realística, enquanto tecnologia aplicada a educação nos cursos de saúde. In: Anais do II Seminário de tecnologias aplicados a educação e saúde; 2015; Salvador.

8. Zahao Z, Niu P, Ji X, Sweet RM. State of simulation in healthcare education: An initial survey in Beijing. Scientific Paper 2017; 21 (1): 1-8.

9. Roman C, Ellwanger J, Becker GC, Silveira AD, Machado CLB, Manfroi WC. Metodologias ativas de ensinoaprendizagem no processo de ensino em saúde no Brasil: uma revisão narrativa. Clinical Biomedical 
Research 2017; 37 (4): 349-357. http://doi.editoracubo.com.br/10.4322/ 2357-9730.73911.

10. Dourado ASS; Giannella TR. Ensino Baseado em Simulação na Formação Continuada de médicos: análise das Percepções de alunos e Professores de um Hospital do Rio de Janeiro.

Rev. Bras de Educação Médica 2014; 38 (4): 460-469.

http://dx.doi.org/10.1590/S010055022014000400007

11. Fernandes AKC; Ribeiro LM; Brasil GC, Magro MCS; Hermann PRS; Ponce-de-Leon CGRM; Viduedo AFS; Funghetto SS. Simulação como estratégia para o aprendizado em Pediatria. REME- Rev Min Enferm 2016; 20 (976): 1-8. DOI: 10.5935/1415-2762.20160046

12. Silva YC; Roquete FF. Competências do gestor em serviços de saúde: análise da produção científica, no período de 2001 a 2011. RAS- Revista de Administração em Saúde 2013; 15 (58): 2-12.

13. Flentje M; Müßel T; Henzel B; Jantzen JP. Simulating a patient's fall as a means to improve routine communication: Joint training for nursing and fifth-year medical students. GMS J Med Educ 2017; 33 (2): Doc19.

14. Sánchez-Ledesma MJ, Juanes JA, Sáncho C, Alonso-Sardón M, Gonçalves J. Acquisition of Competencies by Medical Students in Neurological Emergency Simulation Environments Using High Fidelity Patient Simulators. J Med Syst 2016; 40 (6): 139. DOI 10.1007/s10916-0160496-3

15. Cheng A; Grant V; Auerbach M. Using Simulation to Improve Patient Safety: Dawn of a New Era. JAMA Pediatr 2015; 169 (5): 419-420. doi:10.1001/jamapediatrics.2014.3817.

16. Issenberg SB; Mcgaghie WC; Petrusa ER; Gordon DL; Scalese RJ. Features and uses of high-fidelity medical simulations that lead to effective learning: a BEME systematic review. Medical Teacher 2005; 27 (1): 10-28. https://doi.org/10.1080/014215905000 46924

17. Ilgen J; Sherbino J; Cook D. Technology-enhanced simulation in emergency medicine: A systematic review and meta-analysis. Acad Emerg Med 2013; 20 (2):117-127, 2013. https://doi.org/10.1111/acem.12076 


\section{Anexos}

Tabela 1. Características sociodemográficas dos participantes do estudo $(n=31)$. Belo Horizonte, 2018.

\begin{tabular}{lcc}
\hline \multicolumn{1}{c}{ Variável } & n & $\%$ \\
\hline Sexo & 14 & 45,16 \\
Masculino & 17 & 54,84 \\
Feminino & & \\
Formação & 9 & 29,03 \\
Ensino médio & 20 & 64,52 \\
Superior & 1 & 3,23 \\
Especialização & 1 & 3,23 \\
Não respondeu & & \\
Alunos do Curso & 11 & 100 \\
Gestão de Serviços de Saúde & & \\
Profissionais de saúde & 7 & 35 \\
SAMU & 13 & 65 \\
Corpo de Bombeiros Militar & & 15 \\
Tempo de atuação dos profissionais de saúde & 3 & 20 \\
$\leq 5$ anos & 4 & 25 \\
6 a 10 anos & 5 & 40 \\
$\geq 11$ anos & 8 & \\
Não respondeu & &
\end{tabular}

Fonte: os autores, 2018. 
Tabela 2. Expectativas, assimilação de conteúdo, habilidades e conhecimentos adquiridos durante o simulado $(\mathrm{n}=31)$. Belo Horizonte, 2018.

\begin{tabular}{|c|c|c|c|c|}
\hline Variáveis & $\begin{array}{l}\text { Muito } \\
\text { pouco }\end{array}$ & Pouco & Regular & Muito \\
\hline As expectativas foram atendidas? & - & - & - & $100 \%$ \\
\hline $\begin{array}{l}\text { A utilização e realização de atividades } \\
\text { práticas em cenários reais facilitou sua } \\
\text { assimilação de conteúdo teórico? }\end{array}$ & - & - & - & $100 \%$ \\
\hline $\begin{array}{l}\text { Você adquiriu ou aprimorou conceitos com } \\
\text { a realização desta atividade? }\end{array}$ & - & - & $16,13 \%$ & $83,87 \%$ \\
\hline $\begin{array}{l}\text { Você adquiriu ou aprimorou habilidades } \\
\text { com a realização desta atividade? }\end{array}$ & - & - & $9,68 \%$ & $90,32 \%$ \\
\hline $\begin{array}{l}\text { Acredita ser possível aplicar os } \\
\text { conhecimentos adquiridos durante a } \\
\text { realização dessa atividade em ambiente } \\
\text { real? }\end{array}$ & - & - & $3,23 \%$ & $96,77 \%$ \\
\hline
\end{tabular}

Fonte: os autores, 2018. 


\section{Como citar este artigo}

Silva KR; Martins SM; Farias SMC; Silva AG. A simulação como estratégia de formação de profissionais da saúde. [online], volume 5, n. 1. Editor responsável: Luiz Roberto de Oliveira. Fortaleza, mês e ano, p. 04-15. Disponível em: http://periodicos.ufc.br/resdite/index. Acesso em "dia/mês/ano".

Data de recebimento do artigo: 23/09/2019

Data de aprovação do artigo: 10/02/2020

Data de publicação: 17/04/2020 